\title{
Seudoaneurisma cubital en un niño. Reporte de un caso y revisión bibliográfica
}

\author{
Laura Togneri, Lucas Fernández Korosek \\ Sector de Cirugía de Mano y Reconstructiva de Miembro Superior, Servicio de Ortopedia y Traumatología, Hospital de Pediatría SAMIC \\ “Prof. Dr. Juan P. Garrahan”, Ciudad Autónoma de Buenos Aires, Argentina
}

\begin{abstract}
RESUMEN
Los seudoaneurismas de las arterias de la mano son infrecuentes, se han publicado escasos reportes de casos de adultos, secundarios a un trauma cerrado o abierto. El diagnóstico se confirma mediante ecografía Doppler y otros estudios complementarios. Si no se trata, se puede complicar con trombosis, embolia distal de arterias digitales, lesión nerviosa o necrosis. La cirugía es el tratamiento recomendado y consiste en resección del segmento dañado y ligadura simple, arteriorrafia o reconstrucción vascular con injerto. Los objetivos de este reporte son describir un caso de seudoaneurisma hipotenar secundario a una herida penetrante en un niño y, dada la rareza de este cuadro, revisar, de manera no sistemática, la bibliografía actual.

Palabras clave: Seudoaneurisma; arteria; cubital; mano; tumoración pulsátil.

Nivel de Evidencia: IV
\end{abstract}

\section{Ulnar Artery Pseudoaneurysm in a Child. Case Report and Literature Review}

\section{ABSTRACT}

Pseudoaneurysms of the arteries of the hand are infrequent; few reports of cases in adult patients, secondary to closed or open trauma, have been found in the literature. The diagnosis is confirmed by Doppler ultrasound and other complementary studies. If it is left untreated, the complications include thrombosis, distal digital artery embolism, nerve injury, or necrosis. Surgery is the recommended treatment and consists of resection of the pseudoaneurysm and simple ligation, arteriorraphy, or vascular reconstruction with graft. The objective of this report is to describe a case of hypothenar pseudoaneurysm due to penetrating injury in a pediatric patient, and, due to the rarity of this condition, to conduct a non-systematic review of the current literature.

Keywords: Pseudoaneurysm; artery; ulnar; hand; pulsatile tumor.

Level of Evidence: IV

\section{INTRODUCCIÓN}

El seudoaneurisma o aneurisma falso se define como una dilatación o defecto de la pared arterial que no compromete la totalidad de sus capas, dando lugar a la formación de un hematoma por extravasación, con comunicación del espacio intravascular y extravascular. Su diferencia con el aneurisma verdadero es que compromete las tres capas de la pared arterial. ${ }^{1-3}$

El origen del seudoaneurisma de la arteria cubital, a nivel de la muñeca o distal a ella, varía. Se suele describir al trauma cerrado como parte del síndrome del martillo hipotenar, por percusión repetitiva a este nivel y dilatación de la pared arterial. La lesión penetrante, el cateterismo iatrogénico y el trauma intraoperatorio representan las principales causas invasivas que lo originan. ${ }^{1,4-6}$ Son lesiones infrecuentes, cuyo diagnóstico diferencial incluye lipomas, quistes y tumores sinoviales, abscesos, neurofibromas y sarcomas de partes blandas, entre otros. ${ }^{4} \mathrm{El}$ diagnóstico temprano resulta vital, ya que, si no se lo trata, aumenta el riesgo de trombosis con isquemia distal, compresión y subsecuente lesión nerviosa y necrosis. ${ }^{5,6}$

Recibido el 19-4-2021. Aceptado luego de la evaluación el 1-5-2021 • Dra. LAURA TOGNERI • lauratogneri@hotmail.com

Cómo citar este artículo: Togneri L, Fernández Korosek L. Seudoaneurisma cubital en un niño. Reporte de un caso y revisión bibliográfica.

Rev Asoc Argent Ortop Traumatol 2021;86(5):639-644. https://doi.org/10.15417/issn.1852-7434.2021.86.5.1352 
Se dispone de diversos estudios complementarios para evaluar estas lesiones vasculares. La ecografía Doppler se presenta como la principal alternativa por el bajo costo, su fácil disponibilidad, y porque prescinde de la radiación y la anestesia general en pacientes pediátricos, aunque su desventaja es ser dependiente del operador. Otras opciones incluyen la arteriografía, la resonancia magnética, la angiorresonancia y la angiotomografía. Estos estudios permiten describir la lesión y la ubicación con más detalle, descartar la trombosis y malformaciones vasculares., ${ }^{4,7}$

Los objetivos de este artículo son describir un caso de seudoaneurisma hipotenar secundario a una herida penetrante en un niño y, dada la rareza de este cuadro, revisar, de manera no sistemática, la bibliografía actual.

\section{CASO CLÍNICO}

Varón de 12 años con antecedentes de herida penetrante por vidrio en la región hipotenar de la mano izquierda. En una primera consulta a un centro de atención de tercer nivel, se le realizan una limpieza y la sutura de la piel. En el seguimiento, desarrolló una tumoración renitente, interpretada de origen infeccioso, por lo que le prescribieron antibióticos por vía oral. Tras dos semanas de evolución, el tamaño y el dolor se habían incrementado, por lo que fue derivado a nuestro Servicio. Durante la consulta, refirió dolor 7/10 (escala analógica visual) en la región hipotenar de la mano izquierda. En el examen físico, se observó una tumoración pulsátil ovoide (Figura 1), la prueba de Allen fue positiva, y la movilidad y la sensibilidad distales en el territorio cubital estaban conservadas. En la ecografía Doppler, se detectó, yuxtapuesta a la arteria cubital, una imagen hipoecoica de paredes definidas, contenido líquido interior y signo del yin-yang, clásico del seudoaneurisma (Figura 2). Finalmente, la angiotomografía mostró un seudoaneurisma de la arteria cubital, a nivel del gancho del hueso ganchoso, de 2,17 x 2,45 cm de diámetro (Figura 3).
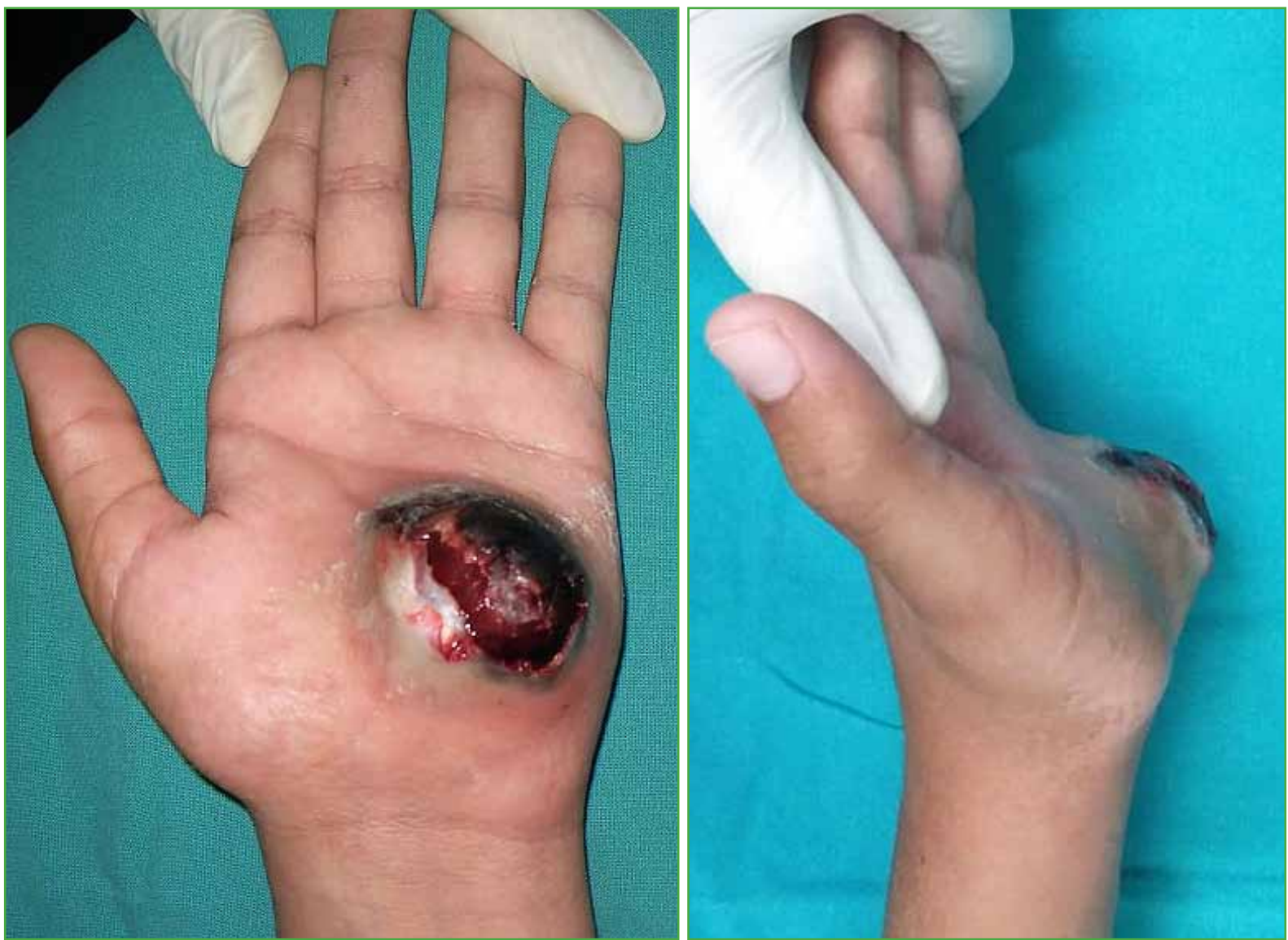

Figura 1. Imagen preoperatoria de frente y de perfil del seudoaneurisma hipotenar. 


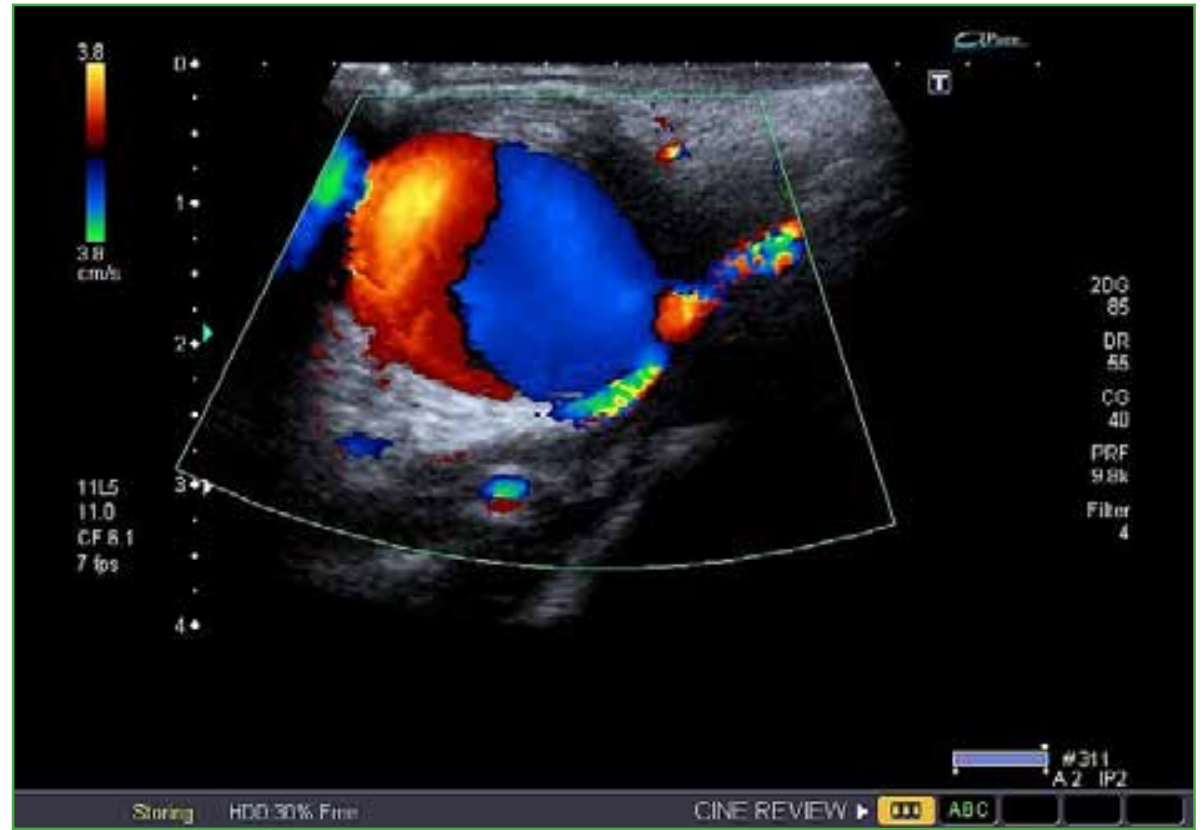

Figura 2. Ecografía Doppler preoperatoria. Se observa el signo del yin-yang, patognomónico del seudoaneurisma.

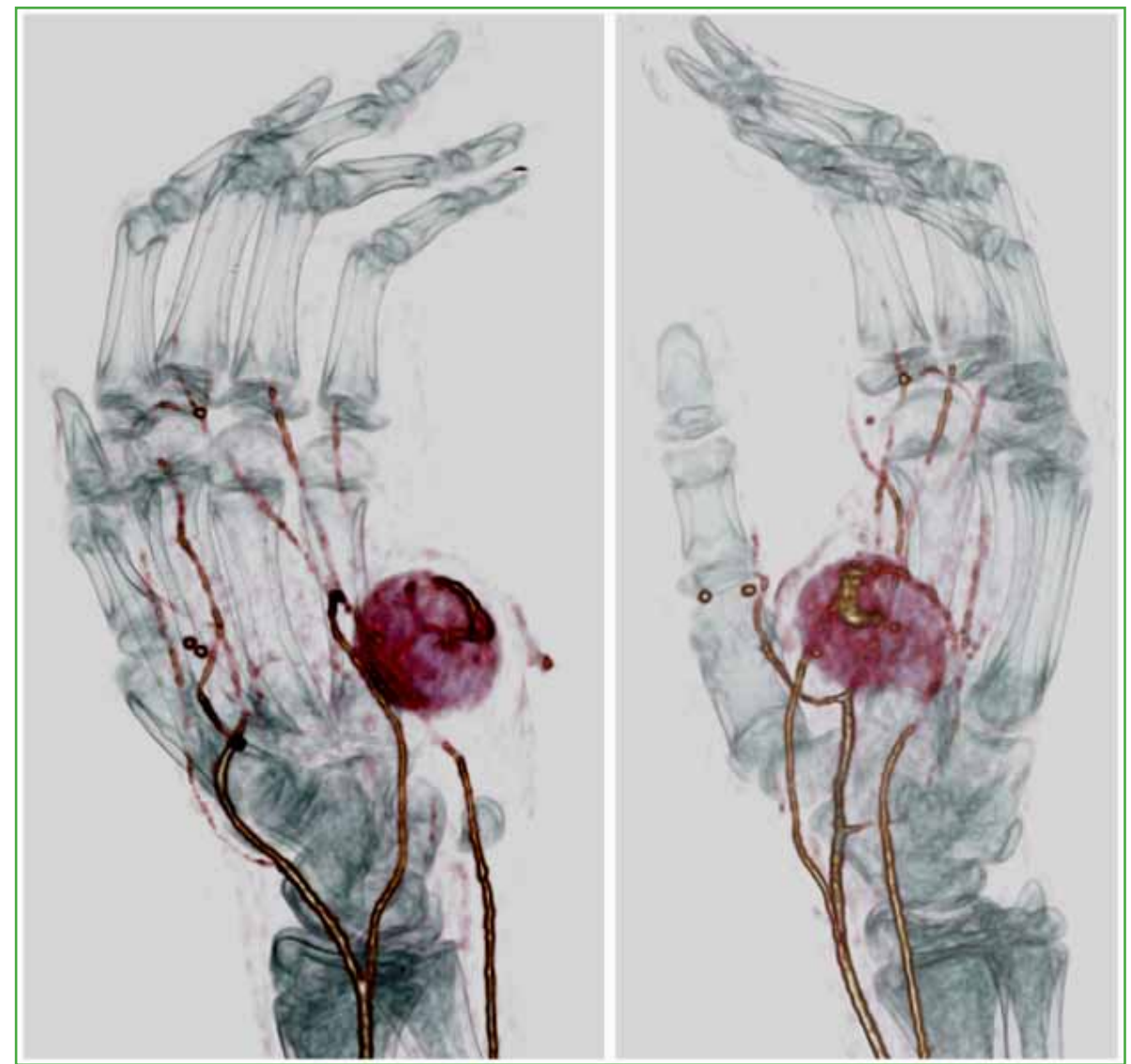

Figura 3. Angiotomografía computarizada preoperatoria con visualización tridimensional del seudoaneurisma hipotenar. 
Luego de confirmar el diagnóstico, el paciente fue sometido a la resección de la tumoración bajo anestesia general y magnificación de 3,5, ampliación a proximal (canal de Guyon) y distal de la herida previa, arteriorrafia cubital y neurólisis microquirúrgica cubital de la rama sensitiva y motora (Figura 4). Se envió la muestra para estudio anatomopatológico. Finalmente, se procedió a colocar un vendaje y una valva antebraquipalmar. Se realizaron controles semanales hasta el retiro de los puntos. Al final del seguimiento, tras seis meses, la herida estaba cicatrizada (Figura 5), el paciente no sentía dolor (0/10), tenía discriminación de dos puntos acral en el territorio cubital de 2 $\mathrm{mm}$ y fuerza de puño de $6 \mathrm{~kg}$. No se registraron complicaciones ni secuelas.

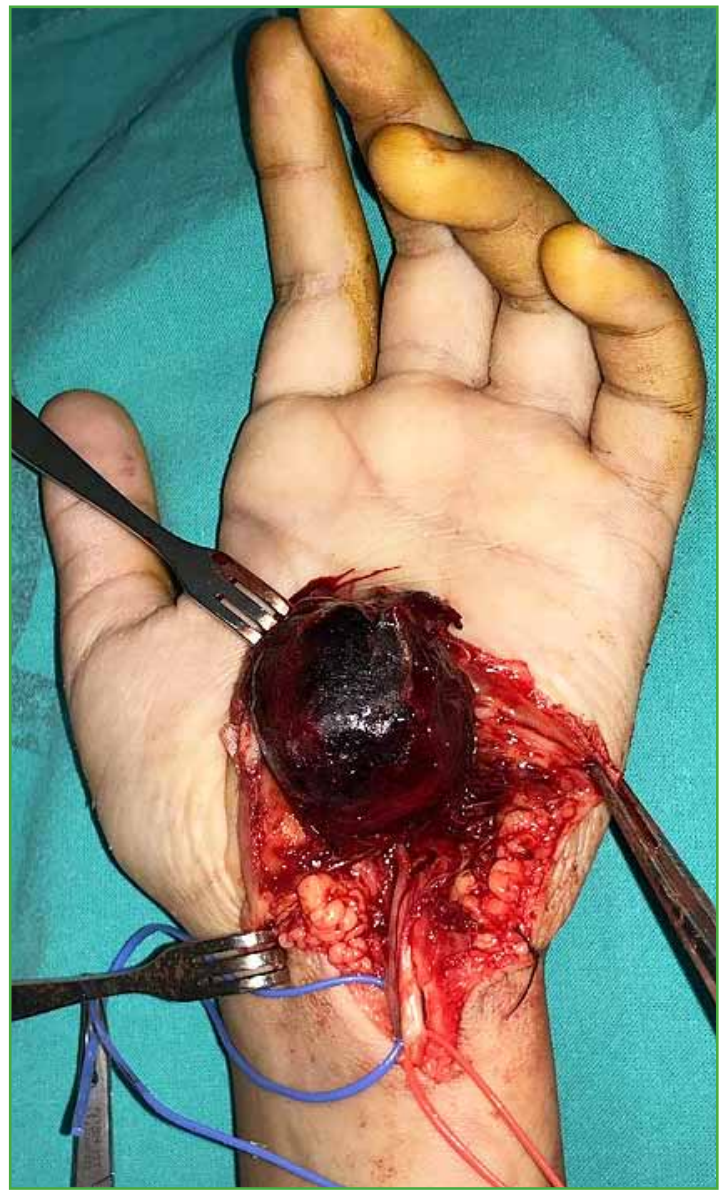

Figura 4. Imagen intraoperatoria del seudoaneurisma hipotenar, con abordaje ampliado a proximal y distal.

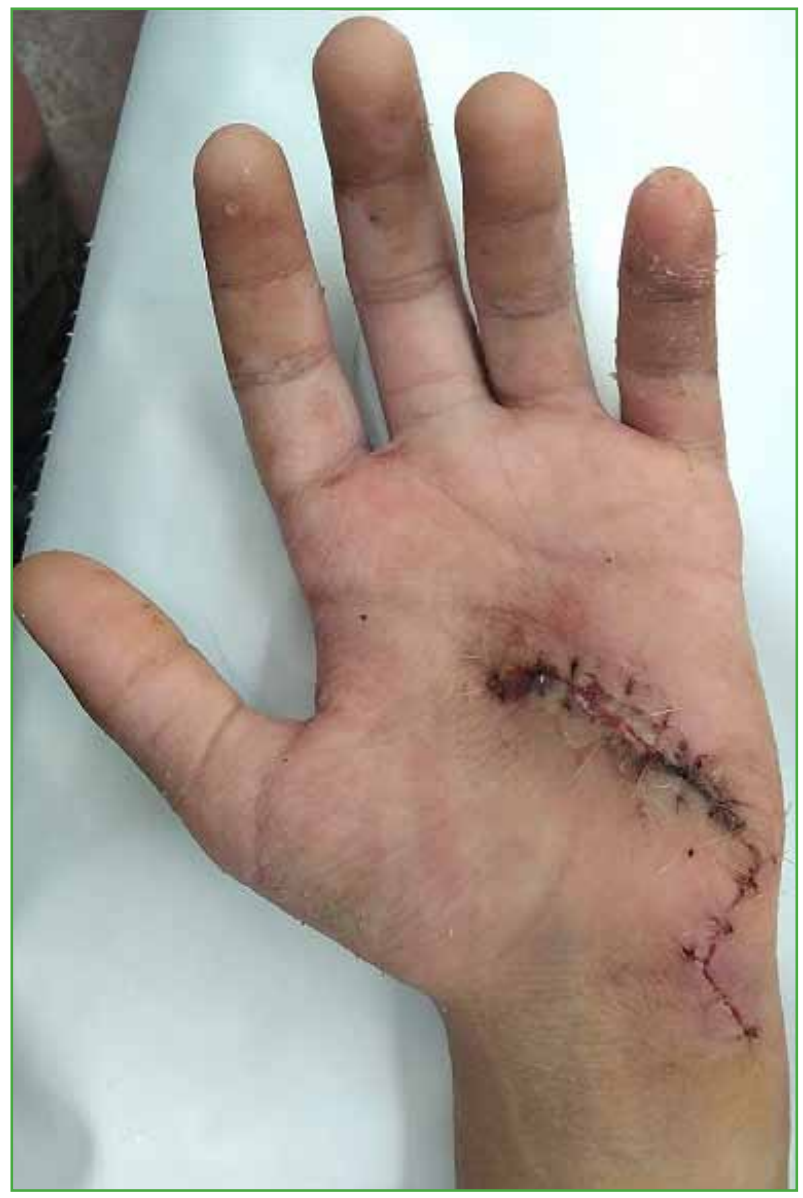

Figura 5. Aspecto a las tres semanas de la resección del seudoaneurisma hipotenar.

\section{DISCUSIÓN}

Debido a la baja incidencia de los seudoaneurismas en la mano, no hay consenso en cuanto a protocolos de tratamiento, las opciones terapéuticas varían entre la resección de la lesión y ligadura de la arteria, y la reconstrucción mediante anastomosis termino-terminal o a través de un bypass venoso. Al constatar la suficiencia vascular solo con la arteria radial mediante la prueba de Allen y estudios complementarios, es factible resecar el seudoaneurisma y ligar los extremos de la arteria sin necesidad de restablecer la circulación mediante un bypass. Sin embargo, esta conducta es discutida en los pacientes pediátricos por el riesgo de sufrir futuras lesiones en un miembro cuya vascularización dependa de un solo vaso. ${ }^{8}$ 
Kalisman y cols. publicaron el caso de un hombre con un falso aneurisma hipotenar luego de un trauma cerrado. El paciente tenía alteración de la sensibilidad en el territorio cubital, aunque sin compromiso de la musculatura intrínseca. Inicialmente se le realizó la resección del falso aneurisma y una anastomosis termino-terminal. A las dos semanas de seguimiento, nuevamente se constató la aparición de una masa pulsátil, se confirmó la recurrencia del falso aneurisma, y se decidió la revisión con resección y ligadura de la arteria cubital, con lo que se logró el alivio definitivo de la signosintomatología. ${ }^{9}$

Dobson y cols., y Hurst y cols. comunicaron casos de seudoaneurisma cubital en el canal de Guyon y en la eminencia hipotenar, respectivamente, secundarios a un trauma penetrante de mano. Además, detectaron hipoestesia secundaria en el territorio cubital y paresia de los músculos intrínsecos de la mano (fuerza 3/5 MRC). Llevaron a cabo la resección del seudoaneurisma y una anastomosis termino-terminal, con una recuperación completa. ${ }^{10,11}$

Thio describió un caso de falso aneurisma cubital hipotenar secundario al uso de un torniquete en una cirugía de resección de un neuroma hipotenar. Se logró un resultado satisfactorio mediante la resección y la anastomosis termino-terminal. ${ }^{12}$

Wang y cols. publicaron la formación de un seudoaneurisma cubital en una mujer añosa, secundario a una fractura de radio y cúbito distal. Con tratamiento incruento de la fractura (debido a la baja demanda funcional), luego de confirmar el flujo colateral distal a cargo de la arteria radial, se procedió a la ligadura de la arteria cubital. ${ }^{13}$

Vancabeke y cols. describieron la formación de un seudoaneurisma cubital coincidente con el sitio de un clavo radial de fijador externo, utilizado como tratamiento definitivo de una fractura expuesta de muñeca. El paciente fue tratado mediante compresión manual y vendaje elástico. Al mes de tratamiento, habían desaparecido los signos clínicos y la ecografía Doppler confirmó la resolución del cuadro, y no hubo recurrencias. ${ }^{14}$

Saour y cols., y González Martínez y cols. presentaron un caso de falso aneurisma que apareció luego de la descompresión convencional de un síndrome del túnel carpiano. Remarcando el emplazamiento cubital del abordaje como problema, inmediatamente después de la cirugía, se detectó una masa pulsátil hipotenar, parestesia en el territorio cubital, dolor con alodinia. Se resecó el saco aneurismático y el alivio del dolor fue inmediato. ${ }^{15,16}$

Unlü y cols. informaron sobre la formación de un seudoaneurisma en el segmento palmar de la arteria cubital, en un paciente con insuficiencia renal crónica. Había sido sometido a una trombectomía venosa por una fístula, con inserción de un catéter de Fogarty hacia la arteria cubital, distal a través de la fístula arteriovenosa. El paciente refirió dolor y una masa pulsátil hipotenar. Finalmente, los síntomas se aliviaron luego de la resección del seudoaneurisma y la anastomosis termino-terminal. ${ }^{17}$

Shaerf y cols., y Komorowska-Timek y cols. informaron la resolución de un seudoaneurisma cubital postraumático en el canal de Guyon, tratado con inyección de trombina guiada por ecografía, asociada a controles seriados para verificar el tamaño del trombo hasta la oclusión completa del falso aneurisma. En este caso, el cuadro se resolvió tras dos procedimientos, confirmado por ecografía, y con función normal de la mano. ${ }^{18,19}$

\section{CONCLUSIÓN}

Sobre la base de la bibliografía revisada, es importante la sospecha diagnóstica del falso aneurisma cubital, en un trauma cerrado o abierto, de variada presentación clínica, con opciones terapéuticas limitadas, para evitar complicaciones o secuelas por falta de tratamiento.

Conflicto de intereses: Los autores no declaran conflictos de intereses. 


\section{BIBLIOGRAFÍA}

1. Erdoes LS, Brown WC. Ruptured ulnar artery pseudoaneurysm. Ann Vasc Surg 1995;9(4):394-6. https://doi.org/10.1007/BF02139413

2. Torres-Blanco Á, Gómez-Palonés F, Ortiz-Monzón E. Rapidly expanding ulnar false aneurysm related to a tablet computer. Ann Vasc Surg 2015;29(5):1015.e5-8. https://doi.org/10.1016/j.avsg.2014.11.010

3. Gimenez DC, Gilabert OV, Ruiz JG, Muns CY, Alter JB, Cubells MD. Ultrasound and magnetic resonance angiography features of post-traumatic ulnar artery pseudoaneurysm: a case report and review of the literature. Skeletal Radiol 2009;38(9):929-32. https://doi.org/10.1007/s00256-009-0715-9

4. Woodley-Cook J, Konieczny M, Simons M. The ulnar artery pseudoaneurysm. BMJ Case Rep 2015;2015:bcr2015212791. https://doi.org/10.1136/bcr-2015-212791

5. Glichenstein J, Ohama J, Lecelrcq C. Tumors of the hand. Berlin: Springer; 1988:147-50.

6. Iyer RS, Hanel DP, Enriquez BK, Weinberger E. Ulnar artery aneurysm causing palmar mass in 5-month-old girl. Pediatr Radiol 2012;42(11):1401-4. https://doi.org/10.1007/s00247-012-2438-7

7. Spittel JA. Aneurysms of the hand and wrist. Med Clin North Am 1958; 42:1007-10. https://doi.org/10.1016/S0025-7125(16)34252-3

8. Sarkar R, Coran AG, Cilley RE. Arterial aneurysms in children: clinicopathologic classification. J Vasc Surg 1991; 13:47-56. PMID: 1987396

9. Kalisman M, Laborde K, Wolff T. Ulnar nerve compression secondary to ulnar artery false aneurysm at the Guyon's canal. J Hand Surg Am 1982;7:137-9. https://doi.org/10.1016/s0363-5023(82)80077-4

10. Dobson P, Purushothaman B, Michla Y, England S, Krishnan MK, Tourret L. Delayed ulnar nerve palsy secondary to ulnar artery pseudoaneurysm distal to Guyon's canal following penetrating trauma to the hand. Ann R Coll Surg Engl 2013;95:e75-e76. https://doi.org/10.1308/003588413X13511609955850

11. Hurst SA, Raveendran S, Eckersley JR. Ulnar artery pseudoaneurysm following single non-penetrating trauma to the hypotenar region. J Hand Surg Eur 2014;39(7):786-8. https://doi.org/10.1177/1753193412462309

12. Thio RT. False aneurysm of the ulnar artery after surgery employing a tourniquet. Am J Surg 1972;123(5):604-5. https://doi.org/10.1016/0002-9610(72)90231-0

13. Wang AA, Strauch RJ, Moore JA. Pseudoaneurysm of the ulnar artery occurring after fracture of the distal radius and ulna: a case report. J Hand Surg Am 1998;23(5):933-7. https://doi.org/10.1016/S0363-5023(98)80176-7

14. Vancabeke M, Heiderich B, Bellens B, Putz P. Pseudoaneurysm of the ulnar artery following use of an external fixator-a case report. Acta Orthop Scand 1999;70(5):522-3. https://doi.org/10.3109/17453679909000994

15. Saour S, Khan U, Feldberg L. Ulnar artery pseudoaneurysm post-carpal tunnel decompression. J Hand Surg Eur 2008;33(3):391-2. https://doi.org/10.1177/1753193408090116

16. González Martínez P, Salazar Agorria A, Bravo Ruiz E, Suárez Tornin MJ, Vega Manrique R. Pseudoaneurisma de arteria cubital después de cirugía de túnel del carpo. Angiología 2012;64(5):220-1. https://doi.org/10.1016/j.angio.2012.03.004

17. Unlü Y, Ceviz M, Polat P. False aneurysm in the palmar segment of the ulnar artery: report of a case. Surg Today 2003;33(2):148-50. https://doi.org/10.1007/s005950300034

18. Shaerf DA, Roberton B, Horwitz M. Traumatic pseudoaneurysm of the ulnar artery treated with ultrasound guided thrombin injection. J Hand Surg Eur 2019;44(6):652-4. https://doi.org/10.1177/1753193419841510

19. Komorowska-Timek E, Teruya T, Abou-Zamzam A, Papa D, Ballard JL. Treatment of the radial and ulnar artery pseudoaneurysms using percutaneous thrombin injection. J Hand Surg Am 2004;29(5):936-42.

https://doi.org/10.1016/j.hsa.2004.05.009 